Check for updates

Cite this: Phys. Chem. Chem. Phys., 2020, 22, 13456

Received 10th May 2020, Accepted 3rd June 2020

DOI: $10.1039 / \mathrm{d} 0 \mathrm{cp} 02545 \mathrm{c}$

rsc.li/pccp

\title{
Interaction of light with a non-covalent zinc porphyrin-graphene oxide nanohybrid $\dagger$
}

\author{
Ewelina Gacka, ${ }^{a}$ Gotard Burdzinski, (D) ${ }^{\mathrm{b}}$ Bronistaw Marciniak, ${ }^{\mathrm{ac}}$ Adam Kubas (D) *d \\ and Anna Lewandowska-Andralojc iD *ac
}

\begin{abstract}
The present study explores the influence of graphene oxide $(\mathrm{GO})$ on deactivation pathways of the excited states of zinc 5,10,15,20-tetrakis(4-(hydroxyphenyl))porphyrin (ZnTPPH). The interaction of light with free ZnTPPH molecules and with ZnTPPH molecules adsorbed on graphene oxide sheets was probed via UV-vis spectroscopy, fluorescence spectroscopy, femtosecond pump-probe technique and nanosecond flash photolysis. Formation of the ground-state ZnTPPH-GO complex in solution was monitored by the red-shift of the porphyrin Soret absorption band. It was found that Stern-Volmer fluorescence quenching can be described in terms of two different quenching regimes depending on the GO concentration. In addition, our comprehensive analysis of the steady-state and time-resolved emission experiments led to the conclusion that the observed quenching was entirely attributable to a static mechanism. Laser flash photolysis showed that the triplet lifetime of the ZnTPPH increased in the presence of GO from $174 \mu \mathrm{s}$ to $292 \mu \mathrm{s}$, which is related to the decrease in the rate constant of a radiationless decay mechanism involving rotation of the peripheral hydroxyphenyl rings of the porphyrin. Femtosecond transient absorption spectroscopy demonstrated the presence of a fast photoinduced electron transfer from the singlet excited state of $\mathrm{ZnTPPH}$ to the GO sheets, as indicated by the formation of a porphyrin radical cation. Quantum chemical calculations were used to gain deeper insights into the nature of the electronically excited states in the free ZnTPPH as well as in the ZnTPPH-GO complex.
\end{abstract}

\section{Introduction}

Graphene based hybrid nanomaterials have attracted broad research attention due to their potential applications in solar energy conversion (photocatalysis and photovoltaics). ${ }^{1-6}$ The photoinduced electron transfer (PET) process in those hybrid materials plays a key role in converting solar light into chemical energy or electricity. ${ }^{7}$ One strategy to fabricate hybrid materials is to functionalize graphene with molecules that are photochemically active. The idea of dye/graphene materials is that graphene acts as electron transporter and supporting matrix for the sensitizer, which in turn expands the light absorption of the nanohybrid into the visible range. However, graphene sheets

\footnotetext{
${ }^{a}$ Faculty of Chemistry, Adam Mickiewicz University, Uniwersytetu Poznanskiego 8 , 61-614 Poznan, Poland. E-mail: alewand@amu.edu.pl

${ }^{b}$ Faculty of Physics, Adam Mickiewicz University, Uniwersytetu Poznanskiego 2, 61-614 Poznan, Poland

'Center for Advanced Technology, Adam Mickiewicz University, Uniwersytetu Poznanskiego 10, 61-614 Poznan, Poland

${ }^{d}$ Institute of Physical Chemistry, Polish Academy of Sciences, Kasprzaka 44/52, 01-224 Warsaw, Poland. E-mail: akubas@ichf.edu.pl

$\dagger$ Electronic supplementary information (ESI) available. See DOI: 10.1039/ d0cp02545c
}

are hydrophobic and tend to aggregate due to $\pi-\pi$ interactions that limit possible functionalization and subsequently applications. On the other hand, graphene oxide (GO), which is a graphene derivative bearing various oxygen-derived functional groups, is hydrophilic and can be applied in photocatalytic systems. ${ }^{8}$ Although the properties of graphene oxide are inferior to those of pure graphene, the modification with various oxygencontaining groups at the basal planes of graphene sheets allows it to form stable aqueous suspensions. This is important for functionalization and further application.

Porphyrins are a well-known group of pigments due to some excellent spectroscopic and electrochemical properties. As planar, electron rich aromatic entities, they possess remarkably high extinction coefficients in the visible region. In addition, their PET ability have caused porphyrins to be widely used as photosensitizers in artificial photosynthetic devices. ${ }^{9}$ Therefore, porphyrins and graphene oxide are appealing units to prepare new nanohybrid composites, which can find application in solar-energy conversion. For any practical application of those hybrid materials, it is crucial that PET from porphyrin to GO takes place. ${ }^{10-14}$ In 2010 Wojcik et al. clearly demonstrated for the first time the occurrence of PET in the hybrid material composed of the cationic porphyrin $(5,10,15,20$-tetra(1-methyl-4-pyridino))porphyrin 
tetra( $p$-toluenesulfonate) (TMPyP) and reduced graphene oxide. ${ }^{12}$ Afterwards, Aly et al. have demonstrated, by applying a series of cationic and neutral porphyrins, that the charge-transfer rate between porphyrins and the graphene carboxylate surface is controlled by both the distance and the electronic coupling between the donor and the acceptor units. ${ }^{13}$ Interestingly, to date several Zn(II) porphyrin/graphene based materials have been applied in photochemical processes. ${ }^{13-20}$ Yuan et al. presented a non-noble metal system for photocatalytic $\mathrm{H}_{2}$ generation that combined $\mathrm{Zn}(\mathrm{II})-5,10,15,20$-tetrakis(4-N-methylpyridyl)porphyrin and RGO with decorated $\mathrm{MoS}_{2}$ as the catalyst. ${ }^{15}$ Yuan et al. have shown that the material that employed Zn(II) porphyrins exhibited much higher hydrogen production efficiency compared to an analogue system where free-base porphyrin was used. ${ }^{15}$ Guo et al. have shown that zinc 5,10,15,20-tetra(4-pyridyl)$21 H, 23 H$-porphine nanostructures, assembled onto GO sheets via a surfactant-assisted method, displayed photocatalytic activity for the degradation of rhodamine B. ${ }^{16}$ It has been also demonstrated that GO sheets can be successfully photoreduced using 5-(4-hydroxyphenyl)-10,15,20-tri(p-tolyl) zinc(II) porphyrin under ambient conditions. ${ }^{19}$

Taking into account the number of literature examples involving non-covalent hybrids of Zn porphyrin and graphene, it is surprising that to date there are no documented cases of zinc 5,10,15,20-tetrakis(4-(hydroxyphenyl))porphyrin (ZnTPPH) non-covalently anchored to GO sheets. This is especially true considering the Zhu group's reported enhanced photocatalytic activity toward hydrogen generation in a system where the free base 5,10,15,20-tetrakis(4-(hydroxyphenyl))porphyrin (TPPH) molecules played the role of a photosensitizer adsorbed on the surface of RGO sheets with Pt nanoparticles acting as the dispersed co-catalyst. ${ }^{20}$ Because of the fact that spectroscopic properties of both the ZnTPPH dye itself as well as its hybrids with graphene oxide have not been studied except one report where absorption and emission spectra of ZnTPPH in acetonitrile were measured, ${ }^{21}$ it is well justified to explore in detail how unbound ZnTPPH interacts with light and how this interaction is affected when the dye is non-covalently anchored to graphene oxide sheets. The main aim of the current study is to describe the nature of interaction between the components as well as to elucidate whether an electron-transfer process occurs between constituents within the nanohybrid material. Herein, we present the preparation of a new non-covalent hybrid of ZnTPPH and graphene oxide (ZnTPPH-GO) (Fig. 1), where the dye molecules are attached to graphene oxide sheets via $\pi-\pi$ interactions. This new material was subjected to a detailed photochemical investigation in $\mathrm{EtOH}-\mathrm{H}_{2} \mathrm{O}(1: 2 \mathrm{v} / \mathrm{v})$ as the solvent. In parallel, light-driven properties of the free dye were studied including the excited state lifetimes and transient absorption spectra of the singlet as well as the triplet excited state. We explored in detail the excited-state deactivation processes of ZnTPPH upon addition of GO. The current investigations, based on steady-state and time-resolved fluorescence experiments and femtosecond transient absorption spectroscopy, demonstrated that a fast photoinduced electron transfer from the singlet excited state of the ZnTPPH to the GO moiety

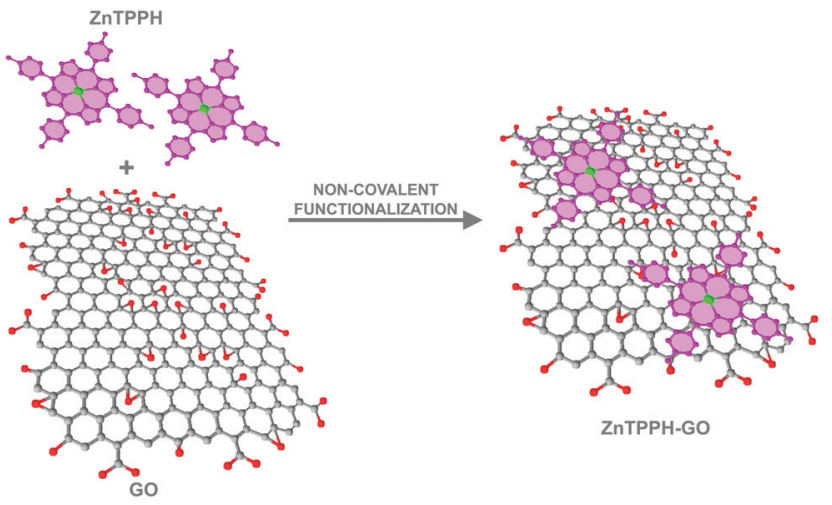

Fig. 1 Illustration of formation of the ZnTPPH-GO nanocomposite via noncovalent functionalization.

occurs in this system. These comprehensive studies coupled to detailed quantum-chemical analysis provide invaluable information that can serve as guidance for fabrication of energy-conversion devices.

\section{Experimental}

\section{Materials}

5,10,15,20-Tetrakis(4-(hydroxyphenyl))porphyrin Zinc(II) (ZnTPPH) and 5,10,15,20-tetrakis(4-(hydroxyphenyl))porphyrin (TPPH) were purchased from Porphyrin Systems, and graphene oxide (GOpowder $<35$ mesh. $\mathrm{C} / \mathrm{O}$ atomic ratio $=2.5-2.6$. Ultrasound bath $1 / 2 \mathrm{~h}$ form perfect single layer dispersion) was purchased from Abalonyx, Ethanol (HPLC grade) was bought from J. T. Baker. Solutions were prepared with ultrapure water. For all experiments GO suspensions were prepared by dispersing GO powder in water followed by ultrasonication for $30 \mathrm{~min}$.

\section{Experimental apparatus}

UV-vis absorption spectra were recorded using a two-beam spectrometer Cary 100 UV-vis scanning from 800 to $200 \mathrm{~nm}$ with $1 \mathrm{~nm}$ increments. Fluorescence spectra were taken on a LS 50B spectrofluorometer (PerkinElmer) (scan speed $240 \mathrm{~nm}$ $\min ^{-1}$, excitation and emission slits $5 \mathrm{~nm}$ ) for solutions with absorbances at the excitation wavelength lower than 0.1. Emission was scanned between 500 and $800 \mathrm{~nm}$ for all of the samples. The fluorescence lifetimes were measured on a Fluorescence Lifetime Spectrometer (FluoTime300 from PicoQuant) with a detection system based on time-correlated singlephoton counting (TCSPC). The emission decay lifetimes were measured following excitation with $440 \mathrm{~nm}$ photodiodes. Samples were placed in $10 \mathrm{~mm}$ quartz cells for all of the steady-state and time-resolved emission measurements. The details of the femtosecond transient absorption spectra have been described elsewhere. ${ }^{22}$ In short, the ultrafast laser system consisted of a short-pulse titanium sapphire oscillator (Mai Tai, Spectra Physics, $70 \mathrm{fs}$ ) followed by a high-energy titanium sapphire regenerative amplifier (Spitfire Ace, Spectra Physics, $100 \mathrm{fs})$. The $800 \mathrm{~nm}$ beam was split into two beams to generate: (1) a pump $\left(\lambda_{\mathrm{exc}}=425,442\right.$, or $\left.455 \mathrm{~nm}\right)$ from the optical 
parametric amplifier (Topas Prime with a NirVis frequency mixer) and (2) probe pulses in the UV-vis range by using sapphire plate (Ultrafast Systems, Helios). The temporal resolution of the setup is about $300 \mathrm{fs}$. For the transient UV-vis measurements a quartz cell with $2 \mathrm{~mm}$ optical path of solution was used with an absorbance of about 0.2 at the excitation wavelength, and the sample solution was stirred by a Teflon-coated bar. The typical pump energy was about $1 \mu \mathrm{J}$. All of the experiments were performed at room temperature. Global analysis of the transient absorption data was made using the Surface Explorer software (Ultrafast Systems). The setup for the nanosecond laser flash photolysis (LFP) experiments and the data acquisition system have been previously described in detail. ${ }^{23}$ LFP experiments were performed using a Nd:YAG laser (532 nm, $5 \mathrm{~mJ}$ ) for excitation. The pulse duration is about $8 \mathrm{~ns}$ and the shortest meaningful lifetime that can be determined from the time evolution of the signal is about 12-15 ns. Transient kinetic profiles were measured at individual wavelengths by the stepscan method in the range of 400 to $750 \mathrm{~nm}$ as the average of 8 pulses. Solutions for LFP were degassed with argon for $15 \mathrm{~min}$ prior to the measurements. Experiments were performed in rectangular quartz cells $(1 \mathrm{~cm} \times 1 \mathrm{~cm})$.

\section{Quantum chemical calculations}

The method of construction and geometry optimization of the graphene oxide model was described earlier. ${ }^{10}$ Briefly, the system is based on the experimental work of Lerf and co-workers $^{24}$ who investigated a structure of GO prepared according to Hummers procedure. ${ }^{25}$ The same procedure was used by the supplier of the GO in the present study. In our model, GO is represented as $\mathrm{C}_{59} \mathrm{O}_{26} \mathrm{H}_{26}$ structure with $\mathrm{C} / \mathrm{O}$ atomic ratio of 2.3 (experimentally 2.5) and contains all chemically relevant functional groups such as epoxy and carboxy groups. The model was optimized within the density functional theory (DFT) using the BP86 functional ${ }^{26,27}$ augmented with $\mathrm{D} \mathrm{BJ}^{27-29}$ and $\mathrm{gCP}^{30}$ corrections to improve the description of weak interactions and to decrease basis set superposition error, respectively. The same setup was used to optimize the ZnTPPH geometry as well as the ZnTPPH-GO nanohybrid assembly. All of the structures were subject to numerical second derivative calculations and were confirmed to possess only positive Hessian eigenvalues. Subsequently, single-point energies were evaluated with the BHLYP functional, ${ }^{31}$ and corresponding $\mathrm{D} 3 \mathrm{BJ} / \mathrm{gCP}$ corrections were added to the total electronic energy. If not stated otherwise, the def2-TZVP basis set $^{32}$ was used throughout the study. Interaction energy between ZnTPPH and GO was calculated as the difference between the energy of the nanohybrid and the energy of the monomers obtained at their relaxed geometries. All of the calculations were performed with the ORCA 4.2.1 suite of programs. ${ }^{33}$

Electronic spectra of the GO, ZnTPPH and ZnTPPH-GO systems were obtained with the simplified time-dependent DFT method (sTD-DFT). ${ }^{34}$ The method allows for efficient calculations of electronic transition energies and transition moments for systems comprising hundreds of atoms and approaches the accuracy of canonical TD-DFT implementations at a fraction of the cost. The method used reference BHLYP orbitals. All of the configuration state functions (CSFs) with energies up to $10 \mathrm{eV}$ above the ground state were considered (EThresh parameter in the ORCA nomenclature). The spectra were simulated by applying Lorenzian broadening with a broadening parameter of $1000 \mathrm{~cm}^{-1}$. In addition, we uniformly red-shifted all of the computed spectra by $950 \mathrm{~cm}^{-1}$ to visually match with the experimental data. In order to analyze the nature of the lowlying excited states, we performed additional multireference calculations using the $n$-electron valence state perturbation theory (NEVPT2) level. ${ }^{35}$ Due to the size of the systems studied, the domain based local pair natural orbital approximation was applied with the default settings (DLPNO-NEVPT2). ${ }^{58}$ Such correlated calculations were based on the state-averaged complete active space self-consistent field (CASSCF) ${ }^{36}$ wave function which allowed us to rigorously treat orbital neardegeneracy present in the porphyrin-based scaffold. The choices of orbitals that build the active spaces were based on occupation numbers of the BHLYP natural orbitals of the respective species. In the case of ZnTPPH, four Gouterman's orbitals ${ }^{37}$ entered the active space along with four electrons and formed a space denoted as CAS $(4,4)$. The ZnTPPH-GO nanohybrid active space included an additional two orbitals of GO and two more electrons. Thus, the final active space in this case was $\operatorname{CAS}(6,6)$. To estimate the relative intersystem crossing probability, the spin-orbit coupling (SOC) matrix elements were evaluated within the quasi-degenerate perturbation theory ${ }^{38}$ as implemented in ORCA.

\section{Results and discussion}

\section{Absorption properties of ZnTPPH}

ZnTPPH is only slightly soluble in water, thus all of the experiments were performed in EtOH- $\mathrm{H}_{2} \mathrm{O}(1: 2 \mathrm{v} / \mathrm{v})$ mixtures. The absorption spectra of ZnTPPH exhibited typical bands expected for metalloporphyrins, i.e., the Soret band arising from the transition to the second excited state $\left(\mathrm{S}_{0} \rightarrow \mathrm{S}_{2}\right)$ and $\mathrm{Q}$ bands corresponding to weak transition to the first excited state $\left(\mathrm{S}_{0} \rightarrow \mathrm{S}_{1}\right)$ which are described by the Gouterman four-electron, four orbital model. ${ }^{39,40}$ As shown in Fig. 2, the UV-vis spectrum of ZnTPPH shows a very strong Soret band with the maximum at ca. $424 \mathrm{~nm}\left(\varepsilon_{424}=5.0 \times 10^{5} \mathrm{M}^{-1} \mathrm{~cm}^{-1}\right)$ and two less intense Q-bands at ca. $559 \mathrm{~nm}$ and $600 \mathrm{~nm}$. These spectra are similar to those reported earlier for ZnTPPH in acetonitrle. ${ }^{21}$ The insertion of $\mathrm{Zn}$ (II) into the porphyrin ring of TPPH caused a small red-shift of the Soret band by $6 \mathrm{~nm}$ together with a decrease in the number of Q-bands. ${ }^{10}$ The decrease in the number of Q-bands in the UV-vis spectra of ZnTPPH in comparison to TPPH is attributed to the increase of the symmetry of the porphyrin ring upon metalation. ${ }^{39,40}$

\section{Absorption properties of ZnTPPH in the presence of GO}

The UV-vis spectroscopy was used to probe the interaction between GO and ZnTPHH in its electronic ground state. UV-vis spectra are illustrated in Fig. 3 for a number of ZnTPPH-GO 


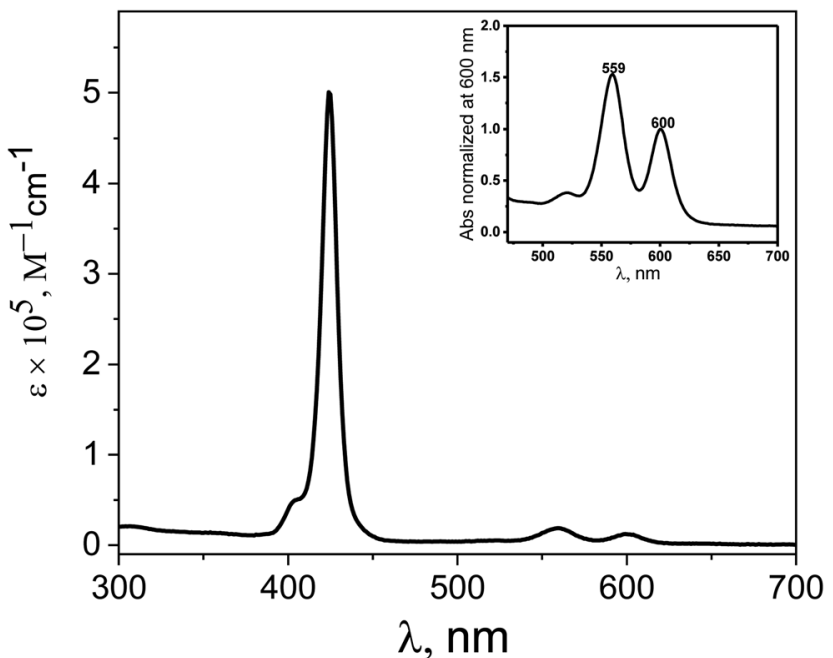

Fig. 2 UV-vis absorption spectra of $3.0 \mu \mathrm{M}$ solution of ZnTPPH in EtOH$\mathrm{H}_{2} \mathrm{O}(1: 2 \mathrm{v} / \mathrm{v})$ (inset: $\mathrm{Q}$-band region of the same spectra).

nanohybrid suspensions measured at a constant ZnTPPH concentration but with a gradual increase of GO. Correction of the UV-vis spectra of ZnTPPH for the GO absorbance revealed that changes in the ZnTPPH absorption are meaningful (inset Fig. 3). Upon adding GO the intensity of the porphyrin's Soret band at $424 \mathrm{~nm}$ gradually decreased, and a new Soret band at $440 \mathrm{~nm}$ arose. An isosbestic point was observed at $433 \mathrm{~nm}$, indicating that there was a clear change from free ZnTPPH to ZnTPPH adsorbed on the surface of GO. The two Q bands were also red shifted. However no formation of the intense band around $700 \mathrm{~nm}$, reported for TPPH and attributed to a partial charge transfer, was observed upon addition of GO. ${ }^{10}$ However theoretical calculations predict that the long-wavelength, charge-transfer band could lie beyond our experimental range i.e. $800 \mathrm{~nm}$ (Fig. 14). However, it can be

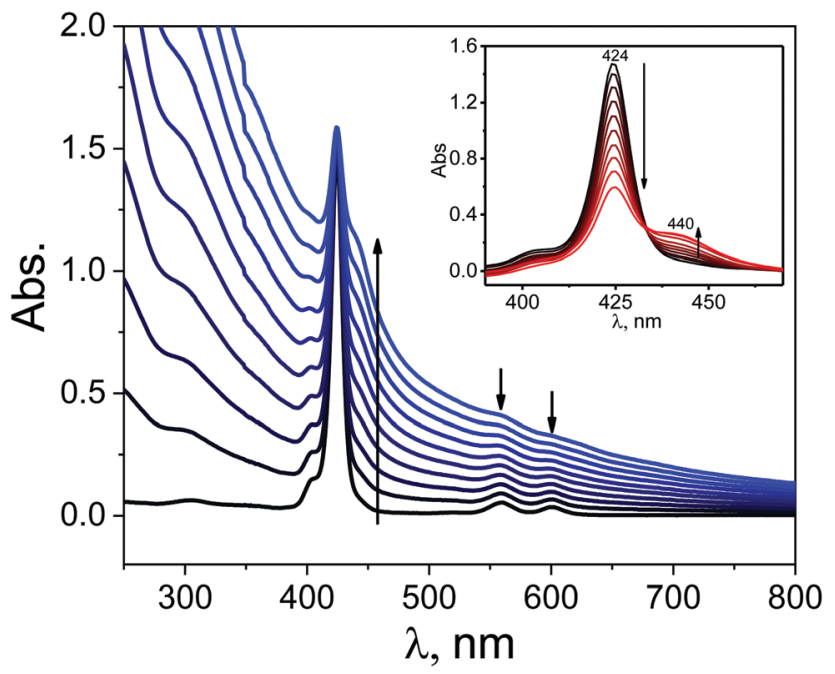

Fig. 3 Absorption spectra recorded during the process of titration of $3 \mathrm{~mL}$ of $3.0 \mu \mathrm{M} \mathrm{EtOH}-\mathrm{H}_{2} \mathrm{O}(1: 2 \mathrm{v} / \mathrm{v})$ solution of $\mathrm{ZnTPPH}$ with $3 \mathrm{mg} \mathrm{mL}^{-1}$ of $\mathrm{GO}$ dispersion $\left(0-0.09 \mathrm{mg} \mathrm{mL}^{-1}\right)$, the inset is the same spectra presented only in the Soret-band region with the spectra corrected for GO absorption. noticed that the Soret band of the $\mathrm{ZnTPPH}-\mathrm{GO}$ exhibited a redshift of $16 \mathrm{~nm}$. The bathochromic shift observed upon nanohybrid formation could be explained by at least two different mechanisms: (1) J-aggregation of the porphyrin molecules, (2) flattening of the porphyrin molecule. The first possibility is highly unlikely because a clear isosbestic point was observed during titration. In addition the linear dependence of the absorbance at the maximum of the Soret band of the nanohybrid on the GO concentration suggests that the obtained material had a well-defined structure. The most plausible explanation for the observed red shift of the Soret band in the ZnTPPH-GO is the considerable distortion in the structure of ZnTPPH upon adsorption on the GO sheet. This interpretation is supported by theoretical calculations (vide infra). The flattening of the porphyrin molecule was also proposed previously in the case of adsorption of TMPyP onto chemically converted graphene $(\mathrm{CCG})^{41}$ or TPPH and GO. ${ }^{10}$

The interaction of ZnTPPH with GO was found to be weaker in comparison to GO nanohybrids with positively charged porphyrins TMAP or TMPyP reported earlier. ${ }^{11,12}$ Even at high GO concentration $0.09 \mathrm{mg} \mathrm{mL}^{-1}$, the Soret band, attributed to free ZnTPPH, unbound to GO, was still present in the UV-vis spectra. It can be explained by weaker $\pi-\pi$ interactions in the current case relative to the strong electrostatic attraction present in the interaction of cationic porphyrin and negatively charged GO.

In order to evaluate the amount of the ZnTPPH that can be adsorbed on $1 \mathrm{mg}$ of GO surfaces, the absorption spectra of GO were measured with the gradual addition of concentrated ZnTPPH solution (Fig. S1, ESI $\dagger$ ). After exceeding the concentration of ZnTPPH above $1.65 \mu \mathrm{M}$, the absorbance of the Soret band at $424 \mathrm{~nm}$ attributed to free porphyrin started to be visible. Based on this experiment, the maximum amount of ZnTPPH adsorbed onto the GO sheets was estimated to be ca. $0.012 \mathrm{mg}$ per $\mathrm{mg}$ of GO. This amount of ZnTPPH adsorbed on the GO surface is almost three times smaller than the analogous value for TPPH, indicating that the introduction of the $\mathrm{Zn}$ (II) into the porphyrin core decreased the strength of the interaction with GO. ${ }^{10}$

Spectroscopic results clearly showed that ZnTPPH interacted with GO in its ground state. The possibility to isolate the newly prepared porphyrin-GO material was examined by a centrifugation experiment controlled by UV-vis spectroscopy. $350 \mathrm{~mL}$ of ZnTPPH solution $\left(4.6 \times 10^{-6} \mathrm{M}\right)\left(\mathrm{EtOH}_{-} \mathrm{H}_{2} \mathrm{O}, 1: 2 \mathrm{v} / \mathrm{v}\right)$ were mixed with $39 \mathrm{~mL}$ of aqueous GO suspension $\left(3 \mathrm{mg} \mathrm{mL}^{-1}\right)$. The mixture was then stirred at room temperature for $2 \mathrm{~h}$, resulting in ZnTPPH-GO hybrids. Subsequently the obtained suspension was centrifuged at $12000 \mathrm{rpm}$ for 90 minutes. The obtained supernatant was light yellow, and the precipitate was dark brown. UV-vis spectra of the suspension of the nanohybrid ZnTPPH-GO were recorded before and after centrifugation (Fig. S2, ESI $\dagger$ ). Based on the minor peak attributed to the porphyrin detected in the suspension, it was estimated that ca. $90 \%$ of the nanohybrid was successfully isolated as the precipitate. Based on that value, it was calculated that the weight content of ZnTPPH in the nanohybrids was $1.0 \%$, which 
is in good agreement with the value obtained in the UV-vis titration experiment (Fig. S1, ESI $\dagger$ ). Interestingly, upon suspending again this precipitate, the resulting spectrum was a reconstruction of the UV-vis spectra of the nanohybrid. This experiment confirms that GO can be successfully non-covalently functionalized with ZnTPPH.

\section{Emission studies}

Emission properties of ZnTPPH in the presence of GO. Fluorescence spectroscopy is one of the most sensitive methods for determining interactions between a dye and a quencher. For this reason the interaction of the singlet excited state of ZnTPPH with the GO sheets was also investigated by emission spectroscopy. It is worth emphasizing that any quantitative analysis of fluorescence data of materials containing graphene is complicated due to several issues such as accounting for any absorption of excitation light or any re-absorption of emitted light by GO. ${ }^{42}$ Another aspect that is often neglected is that, in order to get meaningful results, it is crucial to keep the same absorbance at the excitation wavelength during the emission measurement. ${ }^{42}$ Thus in our measurements, to ensure constant absorbance, the solutions were excited at the isosbestic point i.e. $433 \mathrm{~nm}$.

The studied free porphyrin displayed two emission peaks at $612 \mathrm{~nm}$ and $663 \mathrm{~nm}$ (Fig. 4). This spectral pattern is characteristic for the monomeric porphyrin molecules. The quantum yield of the emission of free ZnTPPH fluorescence was determined to be $5.3 \%$. A decrease in the $\mathrm{ZnTPPH}$ fluorescence intensity was observed with increasing GO concentration (Fig. 4). However, one has to be aware that although fluorescence spectroscopy is a great technique, getting valid data can often be demanding. ${ }^{42}$

Absorption of light by GO sheets can be taken into account by introducing the equation for the inner filter effect. ${ }^{42}$ In our study the correction factor for GO absorption of the excitation light was calculated to be as much as $9.5 \%$ for $0.01 \mathrm{mg} \mathrm{mL}^{-1}$ of

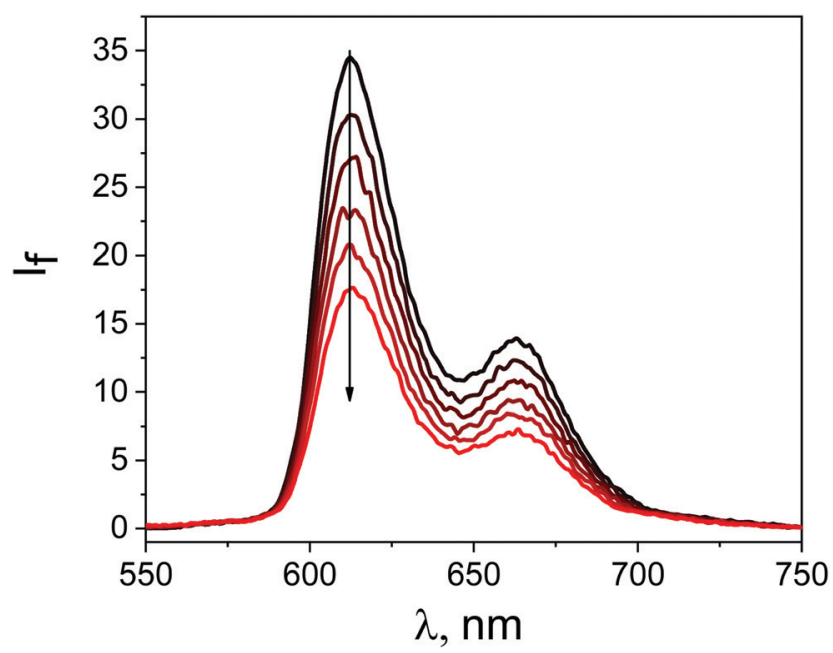

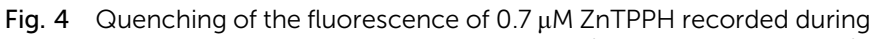
addition of an aqueous suspension of $0.4 \mathrm{mg} \mathrm{mL}^{-1} \mathrm{GO}\left(0-0.01 \mathrm{mg} \mathrm{mL}^{-1}\right)$, $\lambda_{\mathrm{exc}}=433 \mathrm{~nm}$.
GO. A decrease in the ZnTPPH fluorescence intensity was observed with increasing GO concentration (Fig. 5). The quenching efficiency for the GO concentration $0.01 \mathrm{mg} \mathrm{mL}$ was calculated to be $61 \%$. The most interesting observation from the steady-state emission experiment was that the SternVolmer plot $I_{0} / I$ versus the concentration of graphene is not linear. It can be noticed that fitting of the experimental points matches better for with the presence of two different quenching regimes (Fig. 5).

The same quenching behavior was observed for methylene blue, acridine orange and meso-tetra(sulfonatophenyl)porphine in the presence of graphene. ${ }^{43}$ De Miquel et al. rationalized this remarkable behavior as related to the graphene surface area availability to each dye molecule. ${ }^{43}$ The monitored reducing of fluorescence intensity after introducing GO to the ZnTPPH solution (Fig. 5) might be related to the dynamic quenching of an singlet excited state of free ZnTPPH by GO (Fig. 6). The possibility of a dynamic mechanism was probed by recording the emission decay profiles of ZnTPPH in the presence of GO. The fluorescence lifetime was calculated based on a monoexponential fit of the fluorescence decay of ZnTPPH in the absence of GO and was found to be ca. 1.39 ns (Fig. S3, ESI $\dagger$ ). With an increasing amount of GO in the sample, no shortening in the decay profiles of the excited state was noticed. One would normally have expected a shortening of the singlet excited state of unbound ZnTPPH if the quenching were the result of a dynamic process induced by GO. There might also be the chance to see the appearance of a second decay associated with any fluorescence of the nanohybrid. Neither of these decays was detected during the time correlated single photon counting experiment. No measurable change in the singlet excited state lifetime of the free porphyrins excludes the possibility of dynamic quenching by GO (Fig. 5). Our detailed analysis of fluorescence quenching (steady-state and time-resolved) provided

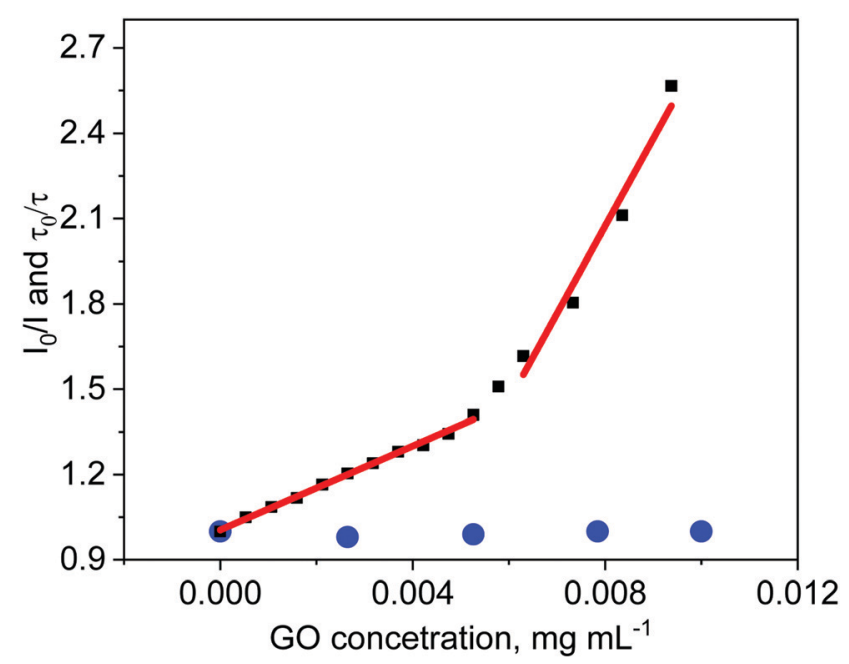

Fig. 5 The relationship between fluorescence intensity $I_{0} / /$ (black symbols) $\left(I_{0}-I_{\mathrm{f}}\right.$ without $\mathrm{GO}, I-I_{\mathrm{f}}$ after addition of $\left.\mathrm{GO}\right)$ and $\mathrm{GO}$ concentration and the relationship between fluorescence lifetime $\tau_{0} / \tau$ (blue symbols) $\left(\tau_{0}\right.$ - fluorescence lifetime without $\mathrm{GO}, \tau$ - fluorescence lifetime after addition of $\mathrm{GO}$ ) and GO concentration for ZnTPPH. Data corrected for inner filter effect. 


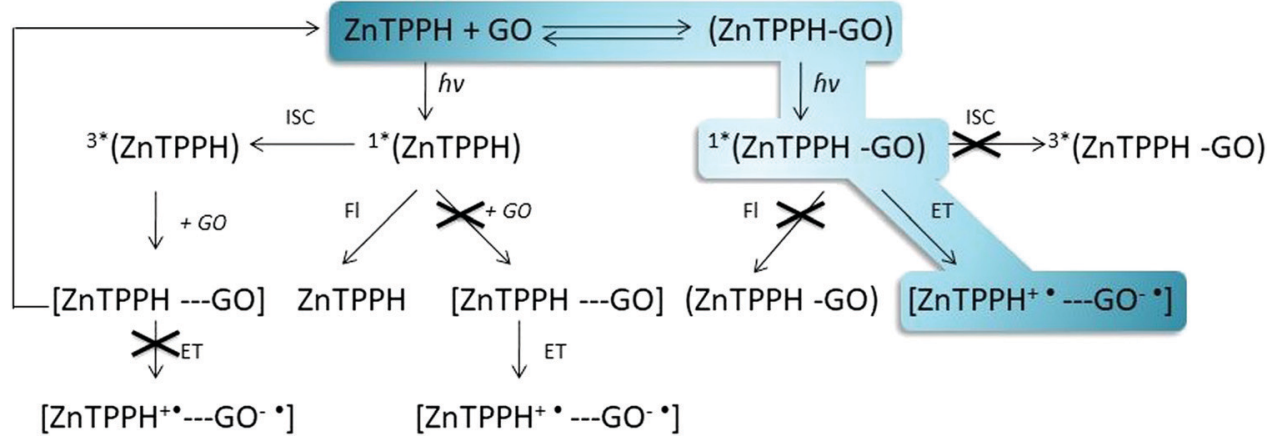

Fig. 6 Pathways of deactivation of excited states of porphyrin ZnTPPH in the presence of graphene oxide.

clear evidence that the observed quenching was entirely attributable to static quenching of the porphyrin by GO.

Numerous papers, dealing with the photophysics of graphene-dye hybrids, interpreted emission data as proof of an electron-transfer process occurring between a dye and graphene, however one cannot identify definitely what is the quenching mechanisms based solely on the steady-state emission experiments. ${ }^{20,44-46}$ Since ZnTPPH-GO has a different electronic structure as found by the changes in its UV-vis absorption spectra compared to the absorption spectrum of unbound ZnTPPH, any emission from the hybrid material would also be expected to be red shifted compared to that of free ZnTPPH. It is important in this case to remark that upon addition of a GO suspension to a ZnTPPH solution, no change in the shape, as well as, in the position of the peaks in the emission spectra was noticed. In addition, as depicted in Fig. 7, the fluorescence excitation spectrum measured for the ZnTPPH solution, after addition of $0.01 \mathrm{mg} \mathrm{mL} \mathrm{m}^{-1}$ of $\mathrm{GO}$, coincided perfectly with the absorption spectrum of free ZnTPPH with a maximum at $424 \mathrm{~nm}$. The results presented above, clearly show that ZnTPPH-GO did not exhibit any measurable emission and

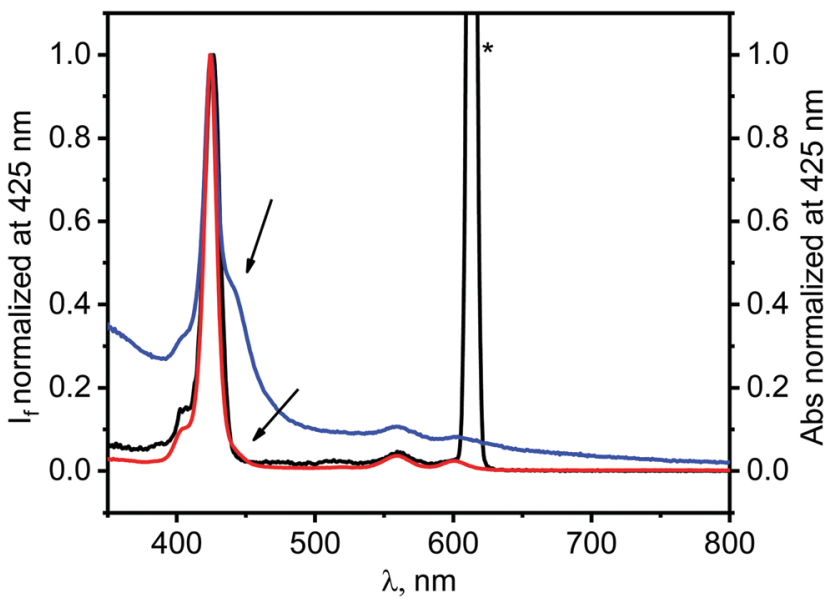

Fig. 7 Normalized fluorescence excitation spectrum measured at $612 \mathrm{~nm}$ of the mixture of ZnTPPH $(0.7 \mu \mathrm{M})$ and $\mathrm{GO}\left(0.01 \mathrm{mg} \mathrm{mL}^{-1}\right)$ (black), absorption spectrum of this mixture (blue) and normalized absorption spectrum of ZnTPPH $(0.7 \mu \mathrm{M})$ in the absence of GO (red). Asterisk denotes scattered excitation light reaching to the detector. that the observed fluorescence in Fig. 4 arose entirely from the free ZnTPPH in the suspension.

The plausible reason for ZnTPPH-GO complex to be nonemissive is the presence of other fast processes that deactivate quickly the singlet excited state.

Femtosecond transient absorption spectroscopy. Femtosecond transient absorption measurements were carried out to confirm that the fast deactivation of the singlet excited state of ZnTPPH in the hybrid ZnTPPH-GO, as expected from the emission experiments, can be attributed to an electron-transfer process in the complex between the photoexcited porphyrin and the graphene oxide sheets. The transient absorption spectra recorded shortly after a $425 \mathrm{~nm}$ laser pulse excitation of ZnTPPH exhibited absorption band with the characteristic spectral shape of singlet excited states of porphyrins (Fig. 8). ${ }^{10,12,13}$ Unbound ZnTPPH in its singlet excited state has a broad, intense absorption band with a maximum at $453 \mathrm{~nm}$ and a Q-band bleach that matches with the Q-band position observed in stationary UV-vis absorption spectra. The excitation wavelength $425 \mathrm{~nm}$ used in the present work corresponds to a transition from $\mathrm{S}_{0}$ to a vibrationally excited level of the $S_{2}$ state of porphyrins. The analysis of transient absorbance kinetics at $455 \mathrm{~nm}$ at short delays

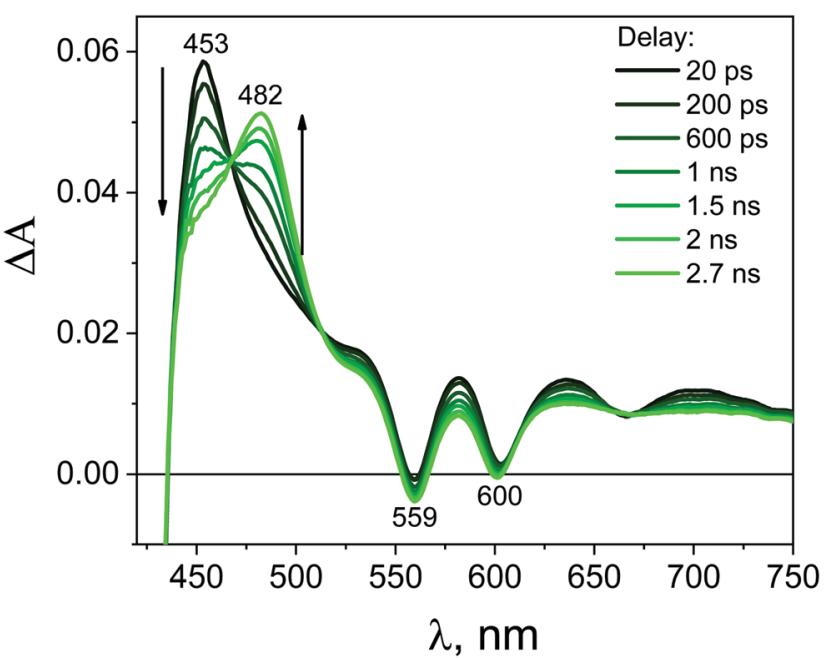

Fig. 8 Transient absorption spectra registered at various time delays for $\mathrm{ZnTPPH}(10.0 \mu \mathrm{M})$ in $\mathrm{EtOH}-\mathrm{H}_{2} \mathrm{O}(1: 2 \mathrm{v} / \mathrm{v})$ following the $425 \mathrm{~nm}$ laser excitation. 
revealed a fast rise component with time constant of only $1.55 \mathrm{ps}$, which can be presumably attributed to the $S_{2} \rightarrow S_{1}$ internal conversion. See Fig. S4 (ESI $\dagger$ ) where the observed growth is that of the $S_{1}$ state which is growing in directly from the decaying $S_{2}$ state. Thus this time constant can be interpreted as the lifetime of the $S_{2}$ excited state. It is in agreement with data reported earlier for other $\mathrm{Zn}$ (II) porphyrins. Using the up conversion fluorescence technique, Gurzadyan et $a l^{47}$ and Mataga et $a l^{48}$ have found a value of $2.3 \mathrm{ps}$ for the lifetime of the $\mathrm{S}_{2}$ state of Zinc(II) tetraphenylporphyrin. Min and co-workers have attributed a transient absorbance decay of 1.6 ps to the $\mathrm{S}_{2} \rightarrow \mathrm{S}_{1}$ transition in $\mathrm{Zn}$ (II) 5,15bis(3,5-dioctyloxyphenyl)porphyrin. ${ }^{49}$ The singlet excited state $\left(\mathrm{S}_{1}\right)$ lifetime of ZnTPPH was calculated from a monoexponential fit to the decay profile at $455 \mathrm{~nm}$ and was found to be $1.47 \mathrm{~ns}$ (Fig. S5, $\mathrm{ESI} \dagger$ ), which is in a good agreement with the data received independently from the TCSPC technique (Fig. S3, ESI $\dagger$ ). As the signal from the ${ }^{{ }^{*}}$ (ZnTPPH) was diminishing (Fig. 8), there was a simultaneous build-up of a strong signal with a maximum at $482 \mathrm{~nm}$ and a low intensity signal in the region $600-750 \mathrm{~nm}$, together with an isosbestic point at $513 \mathrm{~nm}$. The kinetic profile at $482 \mathrm{~nm}$ was fitted to a monoexponential growth function, and the time constant of 1.34 ns was obtained, which is in agreement with the time constant of the signal decay at $455 \mathrm{~nm}$ (Fig. S6, ESI $\dagger$ ) which corresponds to the decay of the $S_{1}$ state population. The broad peak with a maximum absorption at $482 \mathrm{~nm}$ can be identified as the absorption of the ZnTPPH triplet excited state $\left(\mathrm{T}_{1} \rightarrow \mathrm{T}_{n}\right.$ transition) based on the similarity of the transient spectra obtained in the nanosecond flash photolysis experiment (Fig. 11). This assignment is also consistent with the matching of the excited singlet $\left(\mathrm{S}_{1}\right)$ decay of $1.47 \mathrm{~ns}$ into the triplet growth of 1.34 ns. See Fig. S6 (ESI $\dagger$ ) for the matching $\mathrm{S}_{1}$ decay and triplet growth. The intersystem crossing $\mathrm{S}_{1} \rightarrow \mathrm{T}_{1}$ efficiently produces ZnTPPH in the triplet excited state.

After mixing the GO and ZnTPPH solutions in such ratio that all ZnTPPH molecules are adsorbed on the GO surface, the recorded transient absorption (TA) spectra (Fig. 9) varied in comparison to the TA spectra (Fig. 8) of free ZnTPPH. At first, before drawing any conclusions concerning the interaction between ZnTPPH in the singlet excited state and GO, it was of great importance to correct the obtained spectra for the transient absorbance of the GO itself (Fig. S7, ESI $\dagger$ ). ${ }^{50,51}$ Fig. S8 $(\mathrm{ESI} \dagger)$ presents the TA recorded before the correction for the GO transient absorption.

Importantly, the intense signal in the region $450-550 \mathrm{~nm}$ assigned to the singlet excited state absorption of ZnTPPH disappeared. As shown in Fig. 9, a new band in the range of 650-800 $\mathrm{nm}$ was observed. In addition, the small bleach of the two $\mathrm{Q}$ bands could be detected. It seems reasonable to attribute the band $650-800 \mathrm{~nm}$ to the porphyrin cation radical based on the similarity to the reported spectra for the $\mathrm{ZnTPPH}$ radical cation $^{52}$ and the predicted shape of the spectra in the theoretical calculations (vide infra). Neta et al. obtained the spectra of the ZnTPPH radical cation in a pulse radiolysis experiment, and it was found that in neutral solution, the radical cation showed an absorption spectra characterized by a fairly broad band centered at $c a$. $650-690 \mathrm{~nm}$ and a weak shoulder at $760 \mathrm{~nm} .^{52}$

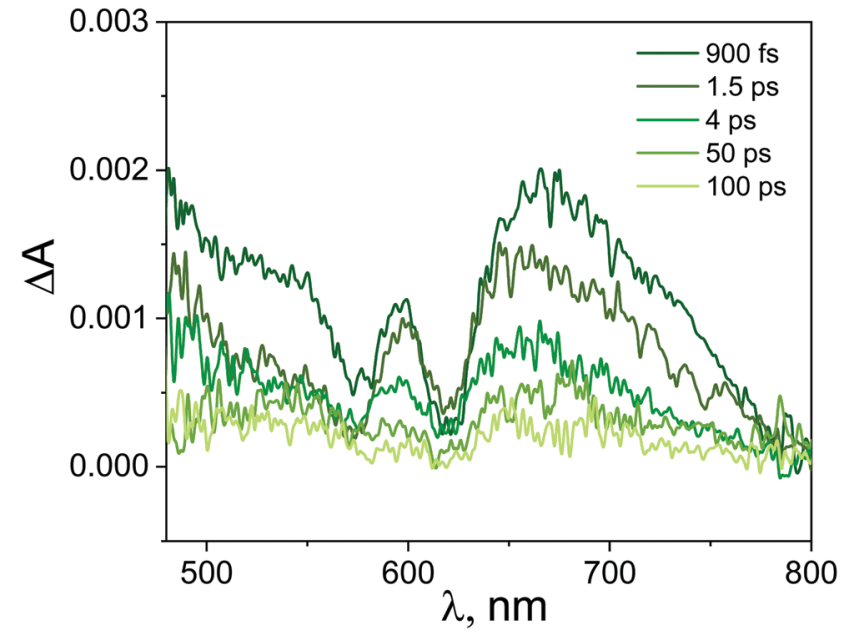

Fig. 9 Transient absorption spectra registered at various time delays for ZnTPPH-GO (ZnTPPH concentration $10.0 \mu \mathrm{M}$, GO concentration $0.4 \mathrm{mg} \mathrm{mL}^{-1}$ ) in water following the $442 \mathrm{~nm}$ laser excitation. Transient absorption spectra were corrected for the contribution from GO itself.

The detection of the radical cation of ZnTPPH provides evidence for a photoinduced electron transfer from the porphyrin to the GO. Interestingly the radical cation of ZnTPPH in our studied system was already detected within the temporal resolution of the instrument (300 fs), which indicates two things: first, electron transfer from the zinc porphyrin to GO is very fast and second that the observed spectra of ZnTPPH radical cation corresponds to vertical electron transfer without relaxation (see theoretical calculations section).

Kinetic profiles recorded for ZnTPPH in the absence and presence of GO are depicted in Fig. 10. The decay of the transient absorption was registered at $700 \mathrm{~nm}$. It is clear that within the same time observation window (50 ps), a significant difference can be seen between the two investigated samples.

The analysis of the kinetic profile obtained for the nanohybrid ZnTPPH-GO is complex due to its similarity to the kinetic profile measured for the GO itself (blue curve in Fig. 10). However, undoubtedly the kinetic profile at $700 \mathrm{~nm}$ for ZnTPPH-GO showed a very fast decay dynamics (two exponential decay) compared with the absence of any decay of the excited state of free porphyrin on the same time scale. The time constants extracted from the decay of the ZnTPPH radical cation are $1.6 \mathrm{ps}$ and $20 \mathrm{ps}$. The slower component is convoluted with the signal from the GO itself. However, the very fast decay of the signal of the ZnTPPH radical cation can be assigned to efficient back electron transfer and recovery of the ground state complex. This is confirmed in transient spectra in Fig. 9, since at delay of 4 ps practically no characteristic ZnTPPH ground state $S_{0}$ depletion is observed.

Nanosecond flash photolysis. Nanosecond transient absorption experiments were performed to probe whether the triplet excited state of ZnTPPH could interact with GO. Upon laser excitation at $532 \mathrm{~nm}, \mathrm{ZnTPPH}$ exhibited a bleaching of the $\mathrm{S}_{0}$ ground state at $425 \mathrm{~nm}, 560 \mathrm{~nm}$ and $600 \mathrm{~nm}$. Those spectral features matched perfectly with the band positions in the 


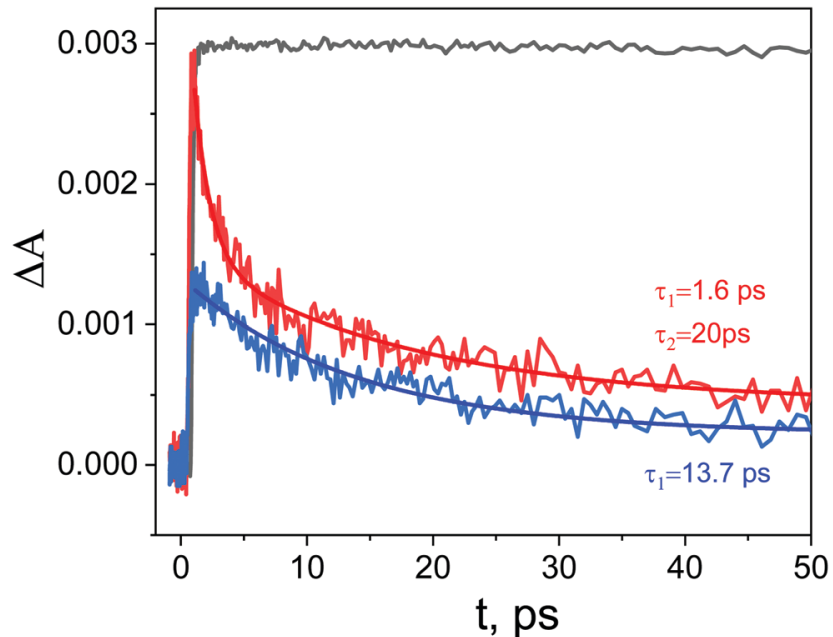

Fig. 10 Transient absorption decays at $700 \mathrm{~nm}$ in $\mathrm{EtOH}-\mathrm{H}_{2} \mathrm{O}(1: 2 \mathrm{v} / \mathrm{v})$ registered for the ZnTPPH (black curve) and ZnTPPH-GO (red curve) and $\mathrm{GO}$ (blue) following the $425 \mathrm{~nm}$ laser excitation for $\mathrm{ZnTPPH}$ and $442 \mathrm{~nm}$ for ZnTPPH-GO and GO. Two exponential decay fit for ZnTPPH-GO (red line) and monoexponential decay fit for GO (blue line).

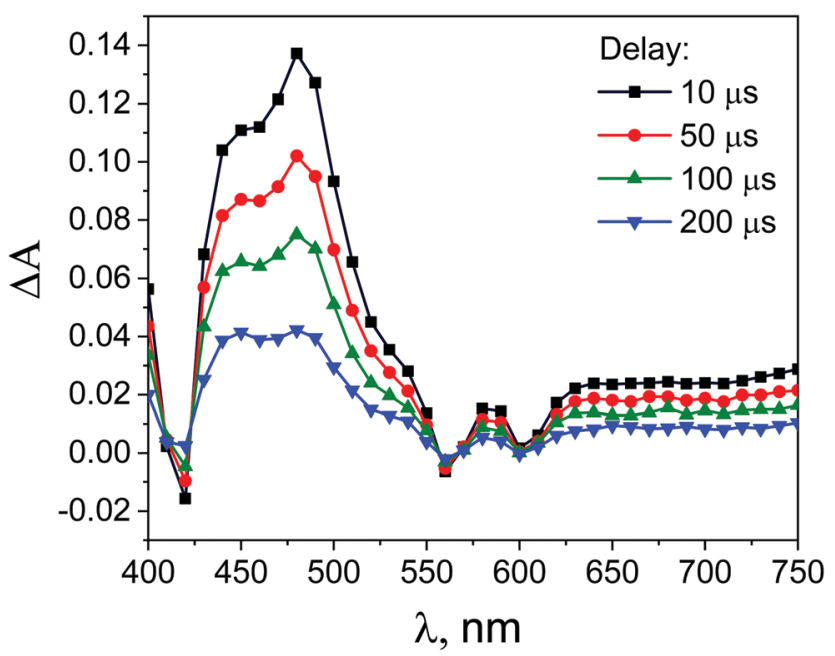

Fig. 11 Transient absorption spectra obtained during laser flash photolysis (with excitation at $532 \mathrm{~nm}$ ) of deoxygenated solutions of ZnTPPH (35 $\mu \mathrm{M})$ in $\mathrm{EtOH}-\mathrm{H}_{2} \mathrm{O}(1: 2 \mathrm{v} / \mathrm{v})$ after different time delays.

ground-state absorption spectrum of ZnTPPH (Fig. 11). The triplet state absorption was observed with a broad, intense band with a maximum at $480 \mathrm{~nm}$ and a weaker band in the range of 600-750 $\mathrm{nm}$ (Fig. 11). The triplet absorption spectrum matched the spectra obtained in the femtosecond transient absorption experiment at the longer time delays (Fig. 8). The triplet lifetime was extracted from a monoexponential fit to the transient decay at $480 \mathrm{~nm}$. The kinetic trace was characterized by the time constant of $174 \mu \mathrm{s}$ (Fig. S9, ESI $\dagger$ ). In order to measure the interaction of the triplet state of the ZnTPPH with the GO, the added amount of GO must be low enough so that free ZnTPPH is still present in the solution. Otherwise if all of the ZnTPPH were adsorbed on the GO surface, fast electron transfer from the singlet excited state of ZnTPPH would prevent intersystem crossing and subsequent population of the triplet state. The intensity of the transient absorption spectra did decrease seven times upon addition of GO $\left(0.02 \mathrm{mg} \mathrm{mL}^{-1}\right)$. This was expected due to part of ZnTPPH being attached to the GO sheet which did not undergo intersystem crossing as a consequence of the very fast process deactivating the singlet excited state when ZnTPPH is complexed to GO (Fig. S10, ESI $\dagger$ ). The intersystem crossing of ZnTPPH and the yield of triplet-state formation was suppressed as observed in other porphyrin-GO hybrids. ${ }^{10,11}$

Surprisingly, the addition of the GO led to the increase of the ZnTPPH triplet lifetime (Fig. S9, ESI $\dagger$ ). The triplet lifetime obtained from the monoexponential fit to the $480 \mathrm{~nm}$ decay profile was found to be as much as $292 \mu \mathrm{s}$. This interesting observation of the increase of the porphyrin triplet lifetime upon addition of graphene has been reported once before in the case of meso-tetra(sulfonatophenyl)porphine. ${ }^{43}$ To explain this somehow unexpected ZnTPPH triplet excited state lifetime increase upon addition of graphene oxide, we propose that in this case the lifetime enhancement is related to conformational restrictions arising when porphyrin molecule in its excited state is adsorbed on the GO surface. Several reports indicate that that triplet excited states decay to the ground state via radiationless mechanisms requires flipping and twisting of conformational free meso-phenyl rings around the core of the porphyrin in which the electronic density of the triplet excited state is mainly located. ${ }^{53,54}$ It was observed that for flexible molecules the triplet lifetimes increase when the molecules are immobilized on a solid surface of cucurbit[n] urils $^{55}$ or in the porous channels of zeolite. ${ }^{56}$ In our case, once the ZnTPPH molecule in the triplet state is adsorbed on the GO sheet, the possibility for the free rotation of the hydroxyphenyl rings, the substituents to the porphyrin core, is largely suppressed. Thus the radiationless deactivation pathway is impeded leading to the observed elongation of the excited state lifetime. The attribution of the increase of the triplet excited state lifetime of ZnTPPH upon adsorption on GO surface to the decrease of the radiationless rate constant were further discussed based on theoretical calculations (vide infra).

Quantum chemical calculations. The orbital picture of free ZnTPPH resembles essentially the Gouterman's picture (Fig. 12): two highest occupied molecular orbitals (HOMOs) are nearlydegenerated similar to the two lowest unoccupied molecular orbitals (LUMOs). The HOMO-LUMO gap of $4.05 \mathrm{eV}$ is marginally larger than in the case of TPPH $(3.93 \mathrm{eV}) .^{10}$

The electronic interaction energy of ZnTPPH with the GO model is relatively large and yields $-22.8 \mathrm{kcal} \mathrm{mol}^{-1}$, comparable to one calculated for TPPH $\left(-22.4 \mathrm{kcal} \mathrm{mol}^{-1}\right) .{ }^{10}$ The optimal distance for this interaction is $4.4 \AA$, marginally shorter than in the case of the TPPH-GO interaction (4.6 $⿱$ ) $)$. The interaction of ZnTPPH with graphene oxide leads to a twisting of the side rings relative to the porphyrin core from about $60^{\circ}$ to $45^{\circ}$, and therefore ZnTPPH gets more flat upon complexation. Analysis of the frontier molecular orbitals of the ZnTPPH-GO nanohybrid, depicted in Fig. 13, reveals a HOMO-LUMO gap of $2.62 \mathrm{eV}$, the value exactly the same like in the case of TPPH. 


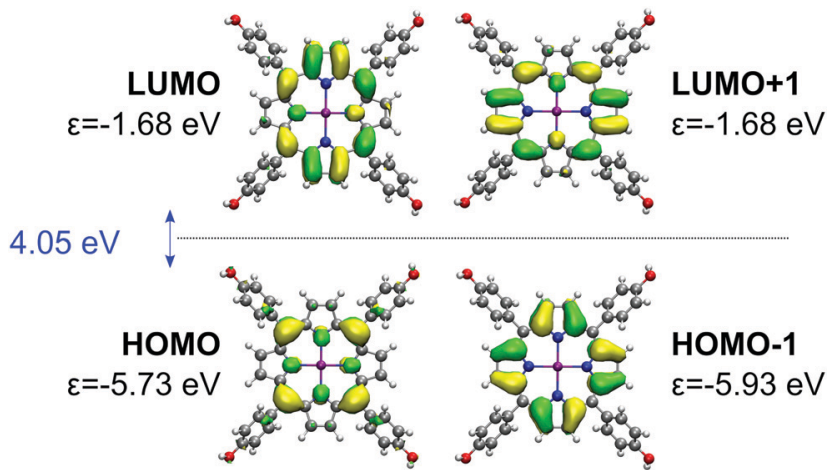

Fig. 12 Gouterman's four orbitals of the ZnTPPH. For each orbital, BHLYP one-electron energy is denoted with $\varepsilon$. HOMO-LUMO gap is given in blue.

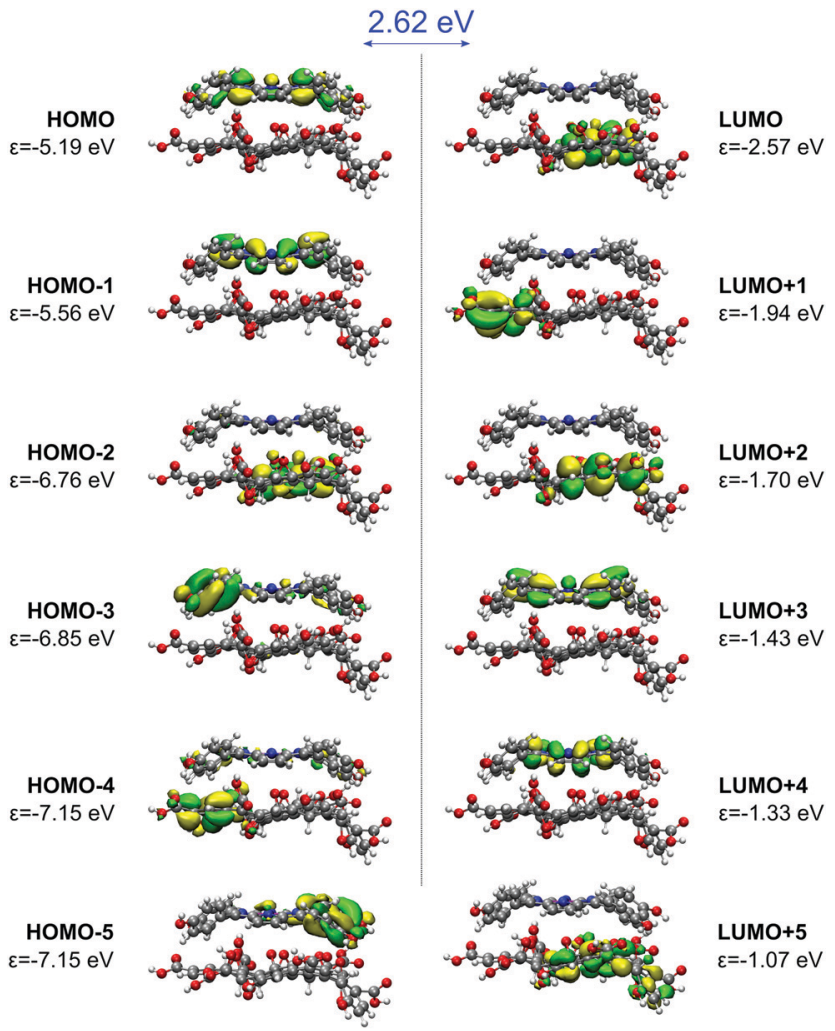

Fig. 13 Frontier molecular orbitals of the ZnTPPH-GO nanohybrid. HOMO-LUMO gap is given in blue.

The HOMO and HOMO-1 orbitals are located within the ZnTPPH moiety while the first three LUMO orbitals are GO-centered. The LUMO +3 and LUMO +4 orbitals correspond to two of Gouterman's $\pi^{*}$ orbitals of the free ZnTPPH.

The presented orbital picture constitutes the basis for the absorption spectra interpretation. Fig. 14 provides a comparison between experimental and sTD-DFT computed spectral lines. The calculations reproduce essentially all non-vibronic transitions. Moreover, the experimentally observed Soret band red-shift of $16 \mathrm{~nm}$ is well reproduced in theory (36 nm). Interestingly, the experimentally broad Soret band has contributions from multiple transitions. As expected, the most intense

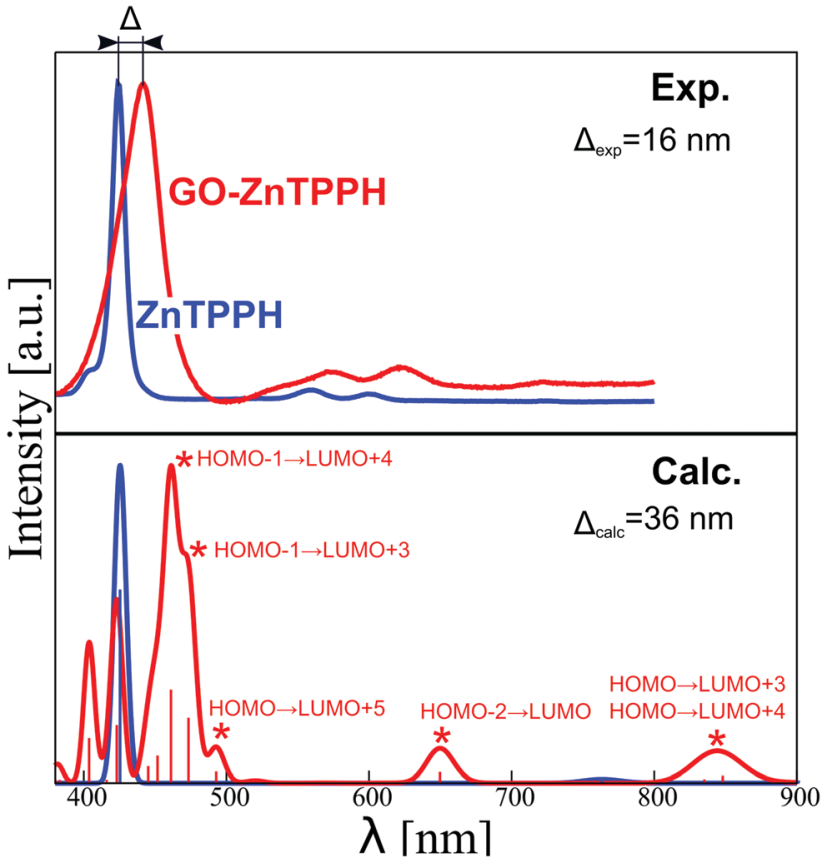

Fig. 14 Comparison of normalized experimental (top) and calculated (bottom) absorption spectra of ZnTPPH (blue) and ZnTPPH-GO complex (red). For the latter, assignment of the observed absorption bands is provided.

are the HOMO-1 $\rightarrow$ LUMO $+3 /+4$ transitions that are within the Gouterman's orbitals system. Interestingly, a lower energy satellite about $490 \mathrm{~nm}$ is of charge-transfer (CT) character from ZnTPPH to GO and involves primarily the HOMO $\rightarrow \mathrm{LUMO}+5$ orbitals.

This CT excited state is presumably responsible for changes in transient absorption spectra recorded with excitation wavelength of $442 \mathrm{~nm}$ in the femtosecond transient absorption spectroscopy. To further confirm the origin of this spectral feature (experiment in Fig. 9), we computed the spectrum of the $\mathrm{ZnTPPH}$ radical cation at two geometries: the geometry of the neutral ZnTPPH and the relaxed, optimal geometry of the radical cation (Fig. S11, ESI $\dagger$ ). The former corresponds to a vertical electron detachment (electron transfer to GO) while the latter involves slow molecular scaffold relaxation. Comparison of simulated and experimental spectra shown in Fig. 9 and Fig. S11 (ESI $\dagger$ ), respectively, undoubtedly proves that the transient absorption bands correspond to ZnTPPH radical cation that did not relaxed to the optimal geometry. This is reasonable taking into account that the ZnTPPH radical cation was detected within the temporal resolution of the instrument (300 fs).

To get further insight into the nature of low-lying excited states of the ZnTPPH-GO nanoassembly, we performed highlevel DLPNO-NEVPT2/CASSCF calculations. The results of these calculations are presented in a form of state-energy diagrams in Fig. 15. As expected, for free ZnTPPH, two degenerated pairs of singlet-excited states $\left(\mathrm{S}_{1} / \mathrm{S}_{2}\right.$ and $\left.\mathrm{S}_{3} / \mathrm{S}_{4}\right)$ are found $1.98 \mathrm{eV}$ and $2.63 \mathrm{eV}$, respectively, above the ground state. The same holds for $T_{1} / T_{2}$ and $T_{3} / T_{4}$ triplet states $(1.70$ and $2.02 \mathrm{eV}$ higher in energy than the ground state, respectively). The computed 


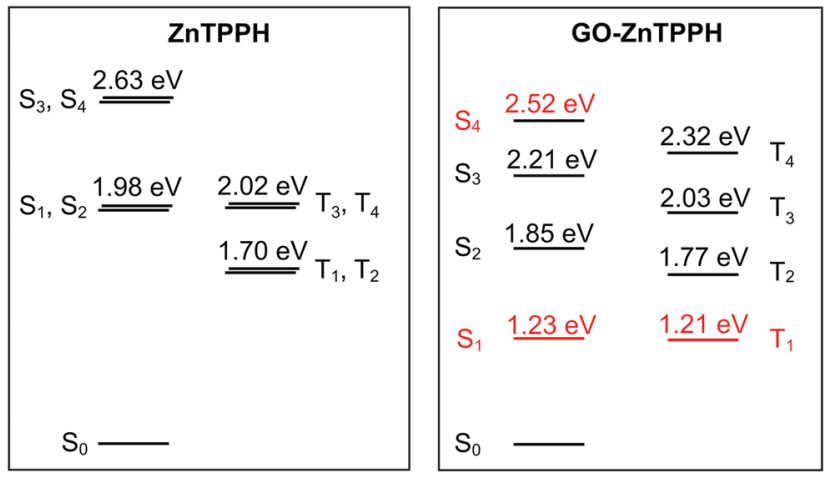

Fig. 15 State-energy diagrams for free ZnTPPH (a) and ZnTPPH-GO nanohybrid (b) computed at the DLPNO-NEVPT2/CASSCF $(4,4)$ and DLPNO-NEVPT2/CASSCF $(6,6)$ levels, respectively. CT states are marked in red. Analysis of dominant configurations for relevant states in the case of GO-ZnTPPH is provided in the ESI, $\uparrow$ Fig. S12.

spin-orbit coupling (SOC) matrix element between $T_{1}$ and $S_{0}$ states is $0.8 \mathrm{~cm}^{-1}$. Once the nanohybrid with GO is formed, the orbital quasi-degeneracy is lifted. Interestingly, the nature of first excited singlet and triplet states changes from porphyrincentered to charge transfer (Fig. S12, ESI $\dagger$ ). Such an $\mathrm{S}_{1}$ CT state was found $1.23 \mathrm{eV}$ above the ground state, but the corresponding $\mathrm{S}_{1} \leftarrow \mathrm{S}_{0}$ transition will gain only marginal intensity ( $f_{\text {osc }} \approx 10^{-4}$ a.u.) as compared to the $S_{2} \leftarrow S_{0}$ transition $\left(f_{\text {osc }} \approx\right.$ 1.6 a.u.). The higher-lying CT state $\left(\mathrm{S}_{4}\right)$ has a markedly larger $f_{\text {osc }}$ of 0.03 a.u. Thus, by tuning the laser excitation to $442 \mathrm{~nm}$, the $\mathrm{S}_{4}$ excited state will gain significant population, and the chargetransfer state would be observed as confirmed experimentally (vide supra).

Flash photolysis experiments showed that the lifetime of the triplet state of the ZnTPPH increased upon complexation with the GO. The SOC matrix elements between $S_{0}$ and all of the triplet states are larger than in the case of free porphyrin ( $>0.8 \mathrm{~cm}^{-1}$, largest element $2.3 \mathrm{~cm}^{-1}$ for $\left\langle\mathrm{S}_{0}\left|\mathbf{H}_{\text {soc }}\right| \mathrm{T}_{2}\right\rangle$ ). The rate constant for decay of the triplet state is proportional to the square of SOC and inversely proportional to energy difference between the involved states at the crossing geometry. ${ }^{57}$ To first approximation, the crossing geometry can be taken as the ground state geometry. The calculated small increase in SOC is thus compensated by the larger difference in energies between $\mathrm{T}_{2}$ and ground $\mathrm{S}_{0}$ states $(\sim 0.07 \mathrm{eV})$ that further underlines the role of relaxation effects that contribute to the elongated lifetime of the triplet state.

\section{Conclusions}

First, in the presented work, we have characterized in detail singlet and triplet excited state properties of ZnTPPH in EtOH$\mathrm{H}_{2} \mathrm{O}(1: 2 \mathrm{v} / \mathrm{v})$. It was determined that the singlet and triplet excited states lifetimes are $1.39 \mathrm{~ns}$ and $174 \mu \mathrm{s}$, respectively. Afterwards, we explored the interaction of GO with the ground state as well as both triplet excited states of ZnTPPH. Formation of the ground-state ZnTPPH-GO complex in solution was confirmed by the red-shift of the porphyrin Soret absorption band as well as the successful isolation of the ZnTPPH-GO nanohybrid material. The computed interaction energy is about $22 \mathrm{kcal} \mathrm{mol}^{-1}$. It was found that Stern-Volmer fluorescence quenching can be described in terms of two different quenching regimes depending on the GO concentration. In addition, our comprehensive analysis of the steady-state and time-resolved emission experiments led to the conclusion that fluorescence quenching was solely attributed to static quenching of the porphyrin by GO. Laser flash photolysis showed that the triplet lifetime of the ZnTPPH was increased in the presence of GO from $174 \mu \mathrm{s}$ to $292 \mu \mathrm{s}$. This lifetime enhancement can be rationalized by the restriction in the rotation of the hydroxyphenyl rings, a motion which is required in the radiationless deactivation of the triplet excited-state mechanism. Surprisingly, fluorescence was not detected for the nanohybrid which indicates that a very fast deactivation process must take place. Ultrafast time-resolved transient absorption spectroscopy demonstrated the occurrence of fast electron transfer from the photoexcited ZnTPPH singlet state to GO sheets, as indicated by the formation of a porphyrin radical cation. The presence of charge-transfer states was also confirmed by our comprehensive theoretical study that also provided detailed insights into the nature of the electronically excited states involved in ZnTPPH-GO photochemistry. These results are relevant to the use of such systems in developing energy-conversion assemblies.

\section{Conflicts of interest}

There are no conflicts to declare.

\section{Acknowledgements}

This research was financially supported by the National Science Centre (project no. 2015/19/D/ST5/00682). A. K. acknowledges support from the National Science Centre, Poland, grant no. 2018/30/E/ST4/00004. Access to high-performance computing resources was provided by the Interdisciplinary Centre for Mathematical and Computational Modelling in Warsaw, Poland, under grants no. GB77-11 and GB79-5. We would like to express our gratitude to Dr G. L. Hug for critically reading the manuscript.

\section{References}

1 J. Albero, D. Mateo and H. García, Molecules, 2019, 24, 906. 2 P. V. Kamat, J. Phys. Chem. Lett., 2011, 2, 242-251.

3 D. Kiessling, R. D. Costa, G. Katsukis, J. Malig, F. Lodermeyer, S. Feihl, A. Roth, L. Wibmer, M. Kehrer, M. Volland, P. Wagner, G. G. Wallace, D. L. Officer and D. M. Guldi, Chem. Sci., 2013, 4, 3085-3098.

4 I. V. Lightcap and P. V. Kamat, Acc. Chem. Res., 2013, 46, 2235-2243.

5 Q. Xiang, B. Cheng and J. Yu, Angew. Chem., Int. Ed., 2015, 54, 11350-11366. 
6 G. Xie, K. Zhang, B. Guo, Q. Liu, L. Fang and J. R. Gong, Adv. Mater., 2013, 25, 3820-3839.

7 A. Arrigo, A. Santoro, M. T. Indelli, M. Natali, F. Scandola and S. Campagna, Phys. Chem. Chem. Phys., 2014, 16, 818-826.

8 D. R. Dreyer, S. Park, C. W. Bielawski and R. S. Ruoff, Chem. Soc. Rev., 2010, 39, 228-240.

9 K. Ladomenou, M. Natali, E. Iengo, G. Charalampidis, F. Scandola and A. G. Coutsolelos, Coord. Chem. Rev., 2015, 304-305, 38-54.

10 E. Gacka, A. Wojcik, M. Mazurkiewicz-Pawlicka, A. Malolepszy, L. Stobiński, A. Kubas, G. L. Hug, B. Marciniak and A. Lewandowska-Andralojc, J. Phys. Chem. C, 2019, 123, 3368-3380.

11 D. Larowska, A. Wojcik, M. Mazurkiewicz-Pawlicka, A. Malolepszy, L. Stobiński, B. Marciniak and A. Lewandowska-Andralojc, ChemPhysChem, 2019, 20, 1054-1066.

12 A. Wojcik and P. V. Kamat, ACS Nano, 2010, 4, 6697-6706.

13 S. M. Aly, M. R. Parida, E. Alarousu and O. F. Mohammed, Chem. Commun., 2014, 50, 10452-10455.

14 D. Masih, S. M. Aly, A. Usman, E. Alarousu and O. F. Mohammed, Phys. Chem. Chem. Phys., 2015, 17, 9015-9019.

15 Y.-J. Yuan, D. Chen, J. Zhong, L.-X. Yang, J.-J. Wang, Z.-T. Yu and Z.-G. Zou, J. Phys. Chem. C, 2017, 121, 24452-24462.

16 P. Guo, P. Chen and M. Liu, ACS Appl. Mater. Interfaces, 2013, 5, 5336-5345.

17 G. Rotas, M. B. Thomas, R. Canton-Vitoria, F. D'Souza and N. Tagmatarchis, Chem. - Eur. J., 2020, 26, 6652-6661.

18 M. B. M. Krishna, V. P. Kumar, N. Venkatramaiah, R. Venkatesan and D. N. Rao, Appl. Phys. Lett., 2011, 98, 081106.

19 M. A. Khaderbad, V. Tjoa, T. Z. Oo, J. Wei, M. Sheri, R. Mangalampalli, V. R. Rao, S. G. Mhaisalkar and N. Mathews, RSC Adv., 2012, 2, 4120-4124.

20 M. Zhu, Z. Li, B. Xiao, Y. Lu, Y. Du, P. Yang and X. Wang, ACS Appl. Mater. Interfaces, 2013, 5, 1732-1740.

21 Y. B. Ivanova, A. S. Parfenov and N. Z. Mamardashvili, Russ. J. Phys. Chem. A, 2017, 91, 94-99.

22 M. Wendel, S. Nizinski, D. Tuwalska, K. Starzak, D. Szot, D. Prukala, M. Sikorski, S. Wybraniec and G. Burdzinski, Phys. Chem. Chem. Phys., 2015, 17, 18152-18158.

23 T. Pedzinski, A. Markiewicz and B. Marciniak, Res. Chem. Intermed., 2009, 35, 497-506.

24 A. Lerf, H. He, M. Forster and J. Klinowski, J. Phys. Chem. B, 1998, 102, 4477-4482.

25 W. S. Hummers and R. E. Offeman, J. Am. Chem. Soc., 1958, 80, 1339.

26 A. D. Becke, Phys. Rev. A: At., Mol., Opt. Phys., 1988, 38, 3098-3100.

27 J. P. Perdew, Phys. Rev. B: Condens. Matter Mater. Phys., 1986, 33, 8822-8824.

28 S. Grimme, S. Ehrlich and L. Goerigk, J. Comput. Chem., 2011, 32, 1456-1465.

29 S. Grimme, J. Antony, S. Ehrlich and H. Krieg, J. Chem. Phys., 2010, 132, 154104.

30 H. Kruse and S. Grimme, J. Chem. Phys., 2012, 136, 154101.
31 A. D. Becke, J. Chem. Phys., 1993, 98, 1372-1377.

32 F. Weigend and R. Ahlrichs, Phys. Chem. Chem. Phys., 2005, 7, 3297-3305.

33 F. Neese, Wiley Interdiscip. Rev.: Comput. Mol. Sci., 2018, 8, e1327.

34 C. Bannwarth and S. Grimme, Comput. Theor. Chem., 2014, 1040-1041, 45-53.

35 C. Angeli, R. Cimiraglia, S. Evangelisti, T. Leininger and J.-P. Malrieu, J. Chem. Phys., 2001, 114, 10252-10264.

36 B. O. Roos, P. R. Taylor and P. E. M. Sigbahn, Chem. Phys., 1980, 48, 157.

37 M. Gouterman, J. Chem. Phys., 1959, 30, 1139-1161.

38 D. Ganyushin and F. Neese, J. Chem. Phys., 2006, 125, 024103.

39 M. Gouterman, J. Mol. Spectrosc., 1961, 6, 138-163.

40 M. Gouterman, G. H. Wagnière and L. C. Snyder, J. Mol. Spectrosc., 1963, 11, 108-127.

41 Y. Xu, L. Zhao, H. Bai, W. Hong, C. Li and G. Shi, J. Am. Chem. Soc., 2009, 131, 13490-13497.

42 A. Lewandowska-Andralojc and B. Marciniak, ACS Energy Lett., 2019, 4, 1898-1901.

43 M. de Miguel, M. Álvaro and H. García, Langmuir, 2012, 28, 2849-2857.

44 Z. Mou, Y. Dong, S. Li, Y. Du, X. Wang, P. Yang and S. Wang, Int. J. Hydrog. Energy, 2011, 36, 8885-8893.

45 W. Zhang, Y. Li and S. Peng, ACS Appl. Mater. Interfaces, 2016, 8, 15187-15195.

46 S. Min and G. Lu, Int. J. Hydrog. Energy, 2012, 37, 10564-10574.

47 G. G. Gurzadyan, T.-H. Tran-Thi and T. Gustavsson, J. Chem. Phys., 1998, 108, 385-388.

48 N. Mataga, Y. Shibata, H. Chosrowjan, N. Yoshida and A. Osuka, J. Phys. Chem. B, 2000, 104, 4001-4004.

49 C.-K. Min, T. Joo, M.-C. Yoon, C. M. Kim, Y. N. Hwang, D. Kim, N. Aratani, N. Yoshida and A. Osuka, J. Chem. Phys., 2001, 114, 6750-6758.

50 S. Kaniyankandy, S. N. Achary, S. Rawalekar and H. N. Ghosh, J. Phys. Chem. C, 2011, 115, 19110-19116.

51 X. Wen, P. Yu, Y.-R. Toh, Y.-C. Lee, K.-Y. Huang, S. Huang, S. Shrestha, G. Conibeer and J. Tang, J. Mater. Chem. C, 2014, 2, 3826-3834.

52 P. Neta, M.-C. Richoux, A. Harriman and L. R. Milgrom, J. Chem. Soc., Faraday Trans., 1986, 82, 209-217.

53 A. Lukaszewicz, J. Karolczak, D. Kowalska, A. Maciejewski, M. Ziolek and R. P. Steer, Chem. Phys., 2007, 331, 359-372.

54 U. Tripathy, D. Kowalska, X. Liu, S. Velate and R. P. Steer, J. Phys. Chem. A, 2008, 112, 5824-5833.

55 C. G. Silva, M. de Miguel, B. Ferrer, M. Álvaro and H. García, Photochem. Photobiol. Sci., 2009, 8, 1650-1654.

56 M. Álvaro, A. Corma, B. Ferrer, H. García and E. Palomares, Phys. Chem. Chem. Phys., 2004, 6, 1345-1349.

57 A. O. Lykhin and S. A. Varganov, Phys. Chem. Chem. Phys., 2020, 22, 5500-5508.

58 Y. Guo, K. Sivalingam, E. F. Valeev and F. Neese, J. Chem. Phys., 2016, 114, 94111. 